\title{
Exploratory factor analysis for small samples
}

\author{
Sunho Jung • Soonmook Lee
}

Published online: 24 March 2011

(C) Psychonomic Society, Inc. 2011

\begin{abstract}
Traditionally, two distinct approaches have been employed for exploratory factor analysis: maximum likelihood factor analysis and principal component analysis. A third alternative, called regularized exploratory factor analysis, was introduced recently in the psychometric literature. Small sample size is an important issue that has received considerable discussion in the factor analysis literature. However, little is known about the differential performance of these three approaches to exploratory factor analysis in a small sample size scenario. A simulation study and an empirical example demonstrate that regularized exploratory factor analysis may be recommended over the two traditional approaches, particularly when sample sizes are small (below 50) and the sample covariance matrix is near singular.
\end{abstract}

Keywords Regularization - Exploratory factor analysis .

Small sample size $\cdot$ Near singular covariance matrix $\cdot$ Monte Carlo simulations

Exploratory factor analysis (EFA) is used for the analysis of interdependencies among observed variables and underlying theoretical constructs, often called factors, so that the underlying structure of observed variables can be discovered. Since its initial development nearly a century ago (Spearman, 1904), EFA has been

S. Jung $(\bowtie)$

Ulsan National Institute of Science and Technology,

School of Technology Management,

Ulsan, Korea

S. Lee

Department of Psychology, SungKyunKwan University,

Seoul, Korea used extensively for a wide variety of behavioral research areas.

In EFA, the issue of sample size has received considerable attention over the decades, because insufficient sample size has often plagued the applications of EFA (MacCallum, Widaman, Zhang, \& Hong, 1999; Tanaka, 1987). The factor analysis literature provides a wide range of rough guidelines regarding an adequate sample size. Most of these guidelines consistently advocate for large samples (say, a sample size of at least 200) to obtain high-quality factor analysis solutions. However, data sets with small samples are frequently encountered in social and behavioral research (MacCallum \& Austin, 2000). In many cases, increasing the sample size may not be possible. In medical research, it is very difficult to collect a large sample of patients suffering from a certain disease. For instance, EFA was applied to specific cortical and subcortical regional brain volume measures from MRI data for 44 schizophrenic patients (Tien et al., 1996). In management studies, sampling units often correspond to firms or products, and thus large sample sizes are hard to obtain.

MacCallum et al. (1999) showed experimentally that the small sample size problem can discourage applied researchers, such as clinical psychologists, from using EFA, particularly under the condition of a low level of communality, which means a higher impact of sample size on factor solution. It has become a serious challenge to use EFA in a situation where the number of observations is less than the dimensionality of each observation, such as in brain imaging data (e.g., Colibazzi et al., 2008).

There exists a vast literature for statistics that describes the importance of regularization, particularly when analyzing high-dimensional data with small sam- 
ples (see Bickel \& Li, 2006, for a detailed overview). Regularization is a useful technique for producing more accurate estimates by the use of prior knowledge. A popular application of regularization is ridge regression (Hoerl \& Kennard, 1970), in which estimates of regression coefficients are shrunk toward zero. The ridge estimates of regression coefficients are, on average, closer to true population values than are their ordinary least squares counterparts.

Conventional EFA involves fitting a factor model to the sample covariance matrix. Due to small samples, the sample covariance matrix tends to be near singular and numerically ill-conditioned, which leads to parameter estimates far from the true parameters. To address the problem with (near) singular covariance matrix in the context of EFA, Finkbeiner and Tucker (1982) proposed using a regularized covariance matrix, which is given by adding a small positive value to the diagonal elements of the sample covariance matrix. This formulation is not entirely new and, methodologically, corresponds to the ridge regression. Fitting the factor model to the regularized covariance matrix was found to give more stable parameter estimates with no risk of nonconvergence (e.g., Yuan \& Chan, 2008).

Traditionally, two distinct approaches have been routinely used for EFA in behavioral research: maximum likelihood factor analysis (MLFA) and principal component analysis (PCA). Recently, a third approach-namely, regularized EFA (REFA) was introduced into the psychometric literature (Jung \& Takane, 2008). This approach is shown to be a viable alternative to MLFA for small samples, in that the number of parameters to be estimated is one.

Very few studies have addressed the role of sample size smaller than 50 in EFA in terms of parameter recovery. de Winter, Dodou, and Wieringa (2009) recently investigated the minimum small sample size necessary to obtain reliable factor solutions under various conditions. They concluded that under the conditions of high communality, high number of observed variables, and small number of factors, EFA can yield stable estimates of population loadings for sample sizes below 50. MacCallum et al. (1999) demonstrated that level of communality is the most important determinant of factor recovery in the issue of sample size. Examining the relative performance of various estimation methods for small samples (below 50) would also be of particular interest to practitioners. Nonetheless, no study has been carried out to investigate the differential performance of various estimation methods in exploratory factor analysis under important conditions such as small sample size and level of communality. Therefore, the objective of this article is to evaluate REFA, MLFA, and PCA in terms of parameter recovery capability on the basis of a Monte
Carlo simulation study in small sample size scenarios. PCA cannot be viewed as a kind of EFA. In the present article, we carry out systematic comparison of the three approaches, because applied researchers have heavily relied on PCA to obtain factor solutions (e.g., Fabrigar, Wegener, MacCallum, \& Strahan, 1999).

The structure of this article is as follows. First, we briefly review regularized EFA and discuss theoretical differences and similarities among the three approaches. Subsequently, we describe the design of our simulation study and report results. Then, an empirical example with a singular covariance matrix is presented to demonstrate the usefulness of REFA. Finally, we summarize the major findings of the simulation study and discuss a number of possible areas of application of REFA in the natural sciences and in medical science and the biosciences.

\section{Background}

There exists a vast literature providing the technical underpinnings of EFA and PCA (e.g., Jolliffe, 2002). Principal component analysis is statistically considered as a way to explore structures in multivariate data by reducing the dimensionality of the data. In the present article, however, PCA is viewed as an estimation method for EFA, as in SPSS, where the sample correlation matrix is eigen-decomposed to produce a factor-loading matrix. PCA does not explicitly model measurement errors. However, measurement errors are ubiquitous in just about any kinds of measurement in psychology. Unlike PCA, EFA explicitly takes into account measurement errors in the observed variables. In EFA, the error variances are subsumed under unique variances, the variabilities in observed variables that are not commonly shared by any other variables.

In EFA, unique variances are usually unknown and must be estimated from the data. Several consistent estimators of unique variances in the factor analysis model have been proposed that do not require iterative procedures and, thus, are efficient computationally (e.g., Hägglund, 1982). They provide estimates of unique variances reasonably close to maximum likelihood estimates (e.g., Ihara \& Kano, 1986). They have been recommended as initial estimates for other iterative methods, such as maximum likelihood (ML), generalized least squares (GLS), and unweighted least squares (ULS) (e.g., Jöreskog \& Sörbom, 1993). Given that unique variances $(\Psi)$ are estimated, factor-loading estimates are obtained by eigen-decomposing $\mathbf{R}-\Psi$ in the ULS and $\mathbf{S}-\Psi$ in the ML and GLS, where $\mathbf{R}$ and $\mathbf{S}$ are sample correlation and covariance matrices, respectively. 
Table 1 The results of an ANOVA test for the mean absolute differences of factor-loading estimates
$\mathrm{SS}$, sum of squares; DF, degrees of freedom; $M S$, = mean square; Sig., significance

\begin{tabular}{|c|c|c|c|c|c|c|}
\hline Source & SS & DF & $M S$ & $F$ & Sig. & $\eta^{2}$ \\
\hline Approach (A) & 27.13 & 2 & 13.56 & $2,159.33$ & .00 & .03 \\
\hline Communality (B) & 47.50 & 2 & 23.75 & $3,780.59$ & .00 & .05 \\
\hline Distribution (C) & 1.25 & 1 & 1.25 & 198.36 & .00 & .00 \\
\hline Sample size (D) & 406.53 & 5 & 81.31 & $12,943.6$ & .00 & .47 \\
\hline$A * B$ & 7.40 & 4 & 1.85 & 294.68 & .00 & .00 \\
\hline $\mathrm{A} * \mathrm{C}$ & .07 & 2 & .03 & 5.28 & .00 & .00 \\
\hline$A * D$ & 37.79 & 10 & 3.78 & 601.53 & .00 & .04 \\
\hline $\mathrm{B} * \mathrm{C}$ & .22 & 2 & .11 & 17.79 & .00 & .00 \\
\hline $\mathrm{B} * \mathrm{D}$ & 1.56 & 10 & .16 & 24.77 & .00 & .00 \\
\hline$C * D$ & .10 & 5 & .02 & 3.25 & .01 & .00 \\
\hline $\mathrm{A} * \mathrm{~B} * \mathrm{C}$ & .27 & 4 & .07 & 10.70 & .00 & .00 \\
\hline$A * B * D$ & 2.67 & 20 & .13 & 21.22 & .00 & .00 \\
\hline$A * C * D$ & .26 & 10 & .03 & 4.20 & .00 & .00 \\
\hline $\mathrm{B} * \mathrm{C} * \mathrm{D}$ & .10 & 10 & .01 & 1.56 & .11 & .00 \\
\hline $\mathrm{A} * \mathrm{~B} * \mathrm{C} * \mathrm{D}$ & .27 & 20 & .01 & 2.14 & .00 & .00 \\
\hline Error & 336.25 & 53,892 & .01 & & & \\
\hline
\end{tabular}

REFA assumes that the true unique variances are proportional to a tentative estimate of unique variances obtained noniteratively. Instead of estimating $\Psi$ as a whole, REFA shrinks these initial estimates with a single parameter $\lambda$ (regularization parameter; see Jung \& Takane, 2008, for the mathematical rationale). Thus, an optimization is done with respect to a single regularization parameter. More specifically, the optimal value of $\lambda$ is chosen in such a way that a discrepancy function associated with ML, GLS, or ULS is minimized. A variety of REFA methods was derived by possible combinations of noniterative estimation methods for unique variances and the three estimation criteria.

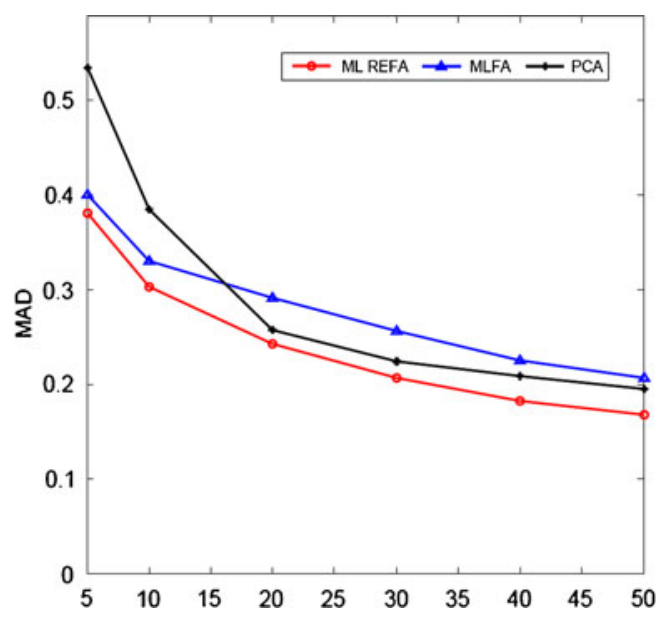

Fig. 1 Average values of the mean absolute differences (MADs) of factor-loading estimates obtained from the three approaches at each level of sample size. ML REFA, one-parameter maximum likelihood estimation method under antiimage; MLFA, maximum likelihood factor analysis; PCA, principal component analysis
Considering a way of estimating unique variances, these variants may be named as the one-parameter family of ULS, GLS, and ML, while the traditional iterative methods
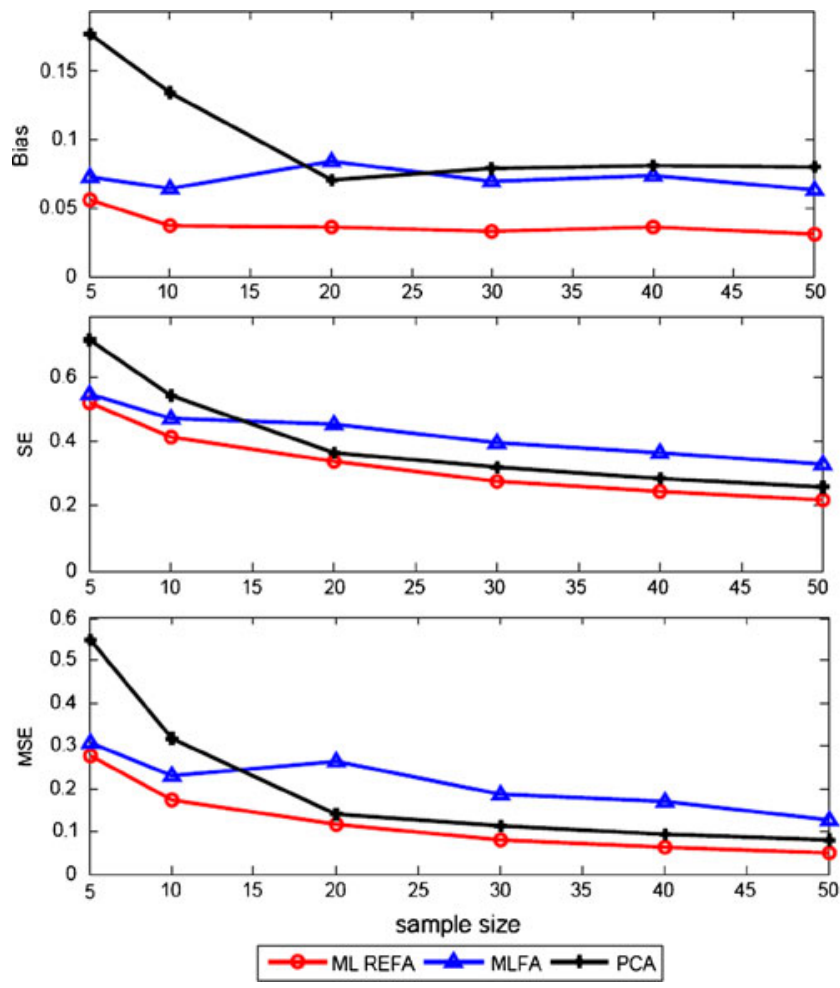

Fig. 2 Overall finite-sample properties of the factor-loading estimates of the three approaches across different sample sizes. SE, standard error; MSE, mean square error; ML REFA, one-parameter maximum likelihood estimation method under antiimage; MLFA, maximum likelihood factor analysis; PCA, principal component analysis 
Table 2 Factor loadings for ML REFA (antiimage assumption) using geomin rotation: Lee's (2008) creativity data (salient factor loadings highlighted in boldface)

\begin{tabular}{|c|c|c|c|c|}
\hline \multirow[t]{2}{*}{ Variable } & \multicolumn{4}{|c|}{ Factor } \\
\hline & 1 & 2 & 3 & 4 \\
\hline 1 & -.33 & -.14 & -.46 & -.02 \\
\hline 2 & -.03 & .65 & -.10 & -.29 \\
\hline 3 & .03 & -.03 & -.73 & -.08 \\
\hline 4 & -.02 & .06 & -.18 & -.48 \\
\hline 5 & -.02 & .56 & .22 & -.15 \\
\hline 6 & -.13 & .07 & -.24 & -.41 \\
\hline 7 & -.31 & -.02 & -.40 & -.60 \\
\hline 8 & -.02 & .30 & -.32 & -.32 \\
\hline 9 & -.38 & .03 & -.11 & -.11 \\
\hline 10 & .02 & .18 & -.42 & -.20 \\
\hline 11 & -.14 & -.04 & -.31 & -.27 \\
\hline 12 & -.33 & .21 & -.25 & .11 \\
\hline 13 & -.26 & -.01 & -.51 & -.05 \\
\hline 14 & -.17 & .04 & -.02 & .24 \\
\hline 15 & -.07 & .40 & -.08 & .17 \\
\hline 16 & .15 & .49 & -.41 & .01 \\
\hline 17 & .12 & .04 & -.82 & .03 \\
\hline 18 & -.17 & .04 & -.72 & .11 \\
\hline 19 & -.46 & .04 & -.14 & .27 \\
\hline 20 & -.26 & .25 & -.23 & .19 \\
\hline 21 & .02 & .42 & -.30 & -.05 \\
\hline 22 & .08 & .69 & .01 & -.13 \\
\hline 23 & .14 & .29 & -.45 & -.14 \\
\hline 24 & .18 & .12 & -.11 & -.33 \\
\hline 25 & .03 & -.08 & -.18 & .05 \\
\hline 26 & -.50 & -.10 & .10 & .36 \\
\hline 27 & .03 & .20 & -.38 & .23 \\
\hline 28 & -.42 & -.06 & -.07 & .33 \\
\hline 29 & -.49 & .00 & -.04 & .41 \\
\hline 30 & -.11 & .53 & -.08 & .24 \\
\hline 31 & -.06 & .11 & -.67 & .10 \\
\hline 32 & -.06 & .27 & -.27 & .20 \\
\hline 33 & -.35 & -.02 & .35 & .23 \\
\hline 34 & -.23 & .18 & -.42 & .10 \\
\hline 35 & -.39 & .26 & -.19 & .17 \\
\hline 36 & .05 & .45 & -.38 & -.03 \\
\hline 37 & -.19 & .04 & -.49 & -.35 \\
\hline 38 & -.10 & .59 & -.08 & -.04 \\
\hline 39 & .03 & .58 & -.26 & -.03 \\
\hline 40 & -.10 & -.04 & -.68 & -.11 \\
\hline 41 & -.05 & .42 & -.38 & -.05 \\
\hline 42 & .15 & .71 & -.08 & .13 \\
\hline 43 & .24 & .44 & -.21 & .07 \\
\hline 44 & .14 & .30 & -.53 & .01 \\
\hline 45 & -.10 & .29 & -.46 & -.11 \\
\hline
\end{tabular}

Table 2 (continued)

\begin{tabular}{lllll}
\hline Variable & \multicolumn{2}{l}{ Factor } & & \\
\cline { 2 - 5 } & 1 & 2 & 3 & 4 \\
\hline 46 & .01 & .31 & -.43 & -.09 \\
47 & .01 & .47 & -.28 & .27 \\
48 & .10 & .49 & -.28 & .05 \\
49 & -.31 & .05 & -.63 & .11 \\
50 & -.24 & .23 & -.49 & .18 \\
51 & -.54 & .27 & .16 & .14 \\
52 & -.58 & .15 & -.19 & .15 \\
53 & -.28 & -.02 & -.38 & .07 \\
54 & .02 & .59 & -.02 & .30 \\
\hline
\end{tabular}

may be named as full ULS, GLS, and ML. Throughout the present article, we use the one-parameter ML estimation method under the antiimage assumption (hereinafter denoted as ML REFA) for comparison with full ML factor analysis (MLFA) and PCA. This particular regularized estimation procedure appears to yield better factor solutions with small samples than do the other combinations (Jung \& Takane, 2008). For the procedure, an initial estimate of unique variances is set equal to variances of antiimage variables, $\operatorname{diag}\left(\mathbf{S}^{-1}\right)^{-1}$.

The permutation tests have been used as a test for identifying the number of significant components in multivariate data analysis techniques (Legendre \& Legendre, 1998). In REFA, permutation tests are employed to estimate the number of factors to retain. These tests are essentially equivalent to parallel analysis described in O’Connor (2000).

\section{Simulation design}

The experimental conditions considered in the present simulation study were as follows: approach (ML REFA, MLFA, and PCA), communality (high, low, and wide), small sample size $(n=5,10,20,30,40$, and 50$)$, and data distribution (normal vs. nonnormal). These experimental conditions are commonly adopted in simulations conducted on factor analysis model (e.g., Curran, West, \& Finch, 1996; MacCallum et al., 1999).

For data generation, three population covariance matrices characterized by 10 variables and three factors were specified that varied with respect to level of communality. These matrices were identical to three of the matrices specified in MacCallum et al. (1999) (see the Appendix). In particular, three levels of communality were defined as follows: high, in which communalities range 
Table 3 Factor loadings for ML REFA (antiimage assumption) using geomin rotation: Lee's (2008) creativity data (salient factor loadings highlighted in boldface)

\begin{tabular}{|c|c|c|c|c|}
\hline \multirow[t]{2}{*}{ Variable } & \multicolumn{4}{|c|}{ Factor } \\
\hline & 1 & 2 & 3 & 4 \\
\hline 55 & -.21 & .02 & -.32 & .42 \\
\hline 56 & -.57 & .15 & .05 & .16 \\
\hline 57 & -.44 & .10 & -.19 & .00 \\
\hline 58 & -.36 & .02 & -.56 & .13 \\
\hline 59 & -.26 & .18 & -.30 & -.08 \\
\hline 60 & -.40 & .33 & .05 & .15 \\
\hline 61 & -.23 & .09 & -.34 & .28 \\
\hline 62 & -.50 & .00 & -.24 & .13 \\
\hline 63 & .04 & .30 & -.16 & .26 \\
\hline 64 & .03 & .32 & -.17 & .07 \\
\hline 65 & -.01 & .06 & -.20 & .43 \\
\hline 66 & -.44 & -.03 & .23 & .27 \\
\hline 67 & -.35 & .04 & -.20 & .18 \\
\hline 68 & -.37 & -.24 & -.13 & .50 \\
\hline 69 & -.27 & .01 & -.40 & .25 \\
\hline 70 & -.14 & -.03 & -.42 & .26 \\
\hline 71 & -.02 & .07 & -.21 & .37 \\
\hline 72 & -.41 & .37 & -.12 & .13 \\
\hline 73 & -.07 & .49 & -.26 & .18 \\
\hline 74 & -.37 & .09 & -.22 & .25 \\
\hline 75 & -.29 & .04 & -.50 & -.04 \\
\hline 76 & -.30 & .44 & -.08 & .23 \\
\hline 77 & .25 & .58 & .04 & -.14 \\
\hline 78 & -.24 & .26 & -.40 & -.14 \\
\hline 79 & -.13 & .58 & -.16 & -.04 \\
\hline 80 & .00 & .48 & -.15 & -.11 \\
\hline 81 & -.16 & .62 & .05 & .06 \\
\hline 82 & -.42 & .29 & -.17 & .00 \\
\hline 83 & -.40 & .18 & -.18 & .01 \\
\hline 84 & -.61 & -.20 & .01 & -.04 \\
\hline 85 & -.19 & .19 & -.08 & -.05 \\
\hline 86 & -.41 & .21 & -.17 & -.22 \\
\hline 87 & -.63 & -.12 & -.41 & -.20 \\
\hline 88 & -.50 & .31 & .18 & -.06 \\
\hline 89 & -.48 & .01 & -.18 & -.06 \\
\hline 90 & -.54 & .05 & -.21 & .02 \\
\hline 91 & -.57 & -.13 & -.18 & -.10 \\
\hline 92 & -.63 & .24 & .29 & -.01 \\
\hline 93 & -.39 & .39 & .03 & -.09 \\
\hline 94 & -.59 & .03 & -.08 & .07 \\
\hline 95 & -.28 & -.06 & -.25 & -.01 \\
\hline 96 & -.45 & .02 & -.03 & .05 \\
\hline 97 & -.34 & .27 & .01 & .21 \\
\hline 98 & -.62 & .30 & .01 & -.14 \\
\hline 99 & -.60 & .01 & .01 & -.10 \\
\hline
\end{tabular}

Table 3 (continued)

\begin{tabular}{|c|c|c|c|c|}
\hline \multirow[t]{2}{*}{ Variable } & \multicolumn{4}{|c|}{ Factor } \\
\hline & 1 & 2 & 3 & 4 \\
\hline 100 & -.28 & -.05 & -.05 & -.35 \\
\hline 101 & -.56 & -.05 & -.01 & -.28 \\
\hline 102 & -.67 & .01 & .32 & -.25 \\
\hline 103 & -.38 & .46 & .44 & -.13 \\
\hline 104 & -.51 & .22 & .02 & -.19 \\
\hline 105 & -.19 & .55 & -.03 & -.03 \\
\hline 106 & -.05 & .63 & -.05 & .05 \\
\hline 107 & -.14 & .20 & -.32 & .04 \\
\hline 108 & -.30 & .61 & .24 & .02 \\
\hline 109 & -.20 & .74 & .38 & .06 \\
\hline
\end{tabular}

from .6 to .7 ; wide, in which communalities took values between .2 and .8 ; low, in which communalities took on small values of .2, .3, and .4. Level of communality can play a vital role in reducing/increasing the impact of sample size on the recovery of the population factors (MacCallum et al., 1999).

Individual-level multivariate normal data were drawn from $N(\mathbf{0}, \boldsymbol{\Sigma})$, where $\boldsymbol{\Sigma}$ is the population covariance matrix, using the Cholesky decomposition (see Wijsman, 1959). To investigate the effect of a nonnormal distribution, we adopted moderate levels of skewness and kurtosis in which skewness was set to 1.25 and kurtosis was set to 3.75 (e.g., Flora \& Curran, 2004). These levels of skewness and kurtosis are quite common in psychological research (e.g., Micceri, 1989). To generate the intended levels of skewness and kurtosis, Fleishman's (1978) power transformation approach was applied to normal data. Five hundred samples were generated at each level of the experimental conditions. Subsequently, all 18,000 sample covariance matrices ( 6 sample sizes $\times 2$ distributions $\times 3$ levels of communality $\times 500$ replications) were fitted by each of the three approaches, which produced factorloading matrices. These factor solutions were unrotated for comparisons of the three approaches. A sample covariance matrix may be near singular due to very small samples, such as 5 and 10. This can lead to factor-loading estimates far from the true parameters. In such an event, the regularized covariance matrix was analyzed using the three approaches.

\section{Simulation results}

To assess the recovery of factor loading under the three approaches, we calculated the mean absolute differences 
(MADs) between parameters and their estimates given by:

$\mathrm{MAD}=\frac{\sum_{\mathrm{j}=1}^{\mathrm{P}}\left|\widehat{\theta}_{\mathrm{j}}-\theta_{\mathrm{j}}\right|}{\mathrm{P}}$,

where $\widehat{\theta}_{\mathrm{j}}$ and $\theta_{\mathrm{j}}$ are an estimate and its parameter, respectively, and $P$ is the number of parameters (e.g., Mason \& Perreault, 1991).

Recovery of parameters An analysis of variance (ANOVA) test was performed that had the MADs of the estimates of loadings as the dependent variable and the four experimental conditions as design factors. Table 1 exhibits the results of the ANOVA test. Most of the main and interaction effects of the design factors turned out to be statistically significant. This is likely to have been due to the huge number of observations (i.e., a total of 54,000 observations). Thus, it is crucial to examine an effect size as well (Maxwell \& Delaney, 1990). We report and interpret those effects whose sizes were close to medium (i.e., $\eta^{2} \approx$ .06) (Cohen, 1988).

Small sample size $\left(\eta^{2}=.47\right)$ and communality $\left(\eta^{2}=\right.$ .05 ) had a large and a medium main effect, respectively. Thus, it is likely that the levels of the MADs of parameter estimates were different between the three levels of communality. Moreover, there are likely substantial differences in the MADs among the six levels of sample size. The MADs tended to decrease with increasing sample size.

Additionally, the two-way interaction effect between small sample size and approach $\left(\eta^{2}=.04\right)$ appeared sizable. Figure 1 displays the average values of the MADs of the three approaches at each level of sample size. As is shown in Fig. 1, REFA had smaller MADs than did the other two approaches across all sample sizes.

The ANOVA test above suggested that the parameter recovery of the three approaches was different among the six levels of sample size. To gain a greater understanding of the behaviors of the three approaches, we further investigated their overall finite-sample properties under the condition of small sample size. With small samples (below 100), low level of communality can cause serious problem, which leads to unstable estimates of parameters (MacCallum et al., 1999). For this reason, sample covariance matrices were first used from the population covariance matrix with a low level of communality. Figure 2 displays the average biases, standard deviations, and mean square errors (MSEs) of parameter estimates obtained from the three approaches. Among these properties, the MSE is the average squared difference between a parameter and its estimate, thereby indicating how far an estimate is, on average, from its parameter; that is, the smaller the MSE, the closer the estimate is to the parameter. Specifically, the $M S E$ is given by

$\operatorname{MSE}\left(\widehat{\theta}_{\mathrm{j}}\right)=\mathrm{E}\left[\left(\widehat{\theta}_{\mathrm{j}}-\theta_{\mathrm{j}}\right)^{2}\right]=\mathrm{E}\left[\left(\widehat{\theta}_{\mathrm{j}}-\mathrm{E}\left(\widehat{\theta}_{\mathrm{j}}\right)\right)^{2}\right]+\left(\mathrm{E}\left(\widehat{\theta}_{\mathrm{j}}\right)-\theta_{\mathrm{j}}\right)^{2}$

This relation indicates that the MSE of an estimate is the sum of its variance and squared bias. Thus, the MSE takes account of both bias and variability of the estimate (Mood, Graybill, \& Boes, 1973).

As is displayed in Fig. 2, ML REFA, on average, yielded unbiased estimates of factor loadings, relative to the other two approaches, across all sample sizes. On the other hand, PCA showed a high level of bias in factorloading estimates, which appeared to increase with sample size. The estimates of ML REFA involved standard deviations smaller than those under the other approaches across all sample sizes. Overall, ML REFA showed smaller MSEs of the estimates of loadings than did the other two over sample size. PCA showed smaller MSEs of loading estimates than did MLFA when the ratio of sample size to the number of observed variables was greater than 1, whereas MLFA yielded smaller MSEs than did PCA when the regularized covariance matrix was factoranalyzed. Figure 2 also showed that MLFA needs roughly four times as many observations $(n=50)$ to achieve the same degree of MSE from ML REFA with $n=15$. A similar pattern of MSE, bias, and variance was also observed with data drawn from population covariance matrices with high and wide communalities. This could be expected because the effect size of the three-way interaction between approach, sample size, and communality was trivial (see Table 1).

\section{Empirical study: an example with a singular covariance matrix}

We next present results from an empirical example to demonstrate the usefulness of REFA with a singular covariance matrix. We used part of the data presented in Lee (2008) in his examination of the structure of workplace creativity. The data set used in this empirical example was obtained using a questionnaire containing a set of 109 items describing a variety of creative behaviors. Among a total of 230 subjects from 23 leading companies, we selected 69 subjects for data analysis, each of whom was proven to be a creative employee by having been awarded a creativity prize. These creative employees adequately represent the intended population. Given the fairly small population of creative employees, we would not consider the data to be insufficient. The creative 
subjects recorded their responses on the basis of how much each of the creativity items described themselves (e. g., I am able to come up with new ideas for solving a longstanding problem) using 4-point scales (i.e., $1=$ not at all, and $4=$ very much).

The data with small samples and large measured variables led to a singular sample covariance matrix. The condition number for the sample covariance matrix in this analysis was $3.8 \times 10^{18}$, which raised serious concern about singularity. To tackle this difficulty, we fit the EFA model to the regularized covariance matrix (e.g., Yuan \& Chan, 2008). This covariance matrix was analyzed using the two approaches: MLFA and ML REFA (i.e., oneparameter maximum likelihood estimation method under the antiimage assumption in the present article). PCA was not used for this particular example because a simulation study reported in the present article showed its poor performance on recovery of population factors with a very small sample size less than the number of observed variables.

For ML REFA, the optimal value of regularization parameter $(\lambda)$ was 0.895 . Permutation tests suggested that four factors were significant. The four factors were then rotated using oblique geomin rotation (Yates, 1984). The factor loadings produced by the ML REFA are presented in Tables 2 and 3. Factor loadings for the factors are considered meaningful if above a threshold of 0.35 . In this particular example, MLFA did not yield a converged solution, both with the sample covariance matrix and with the regularized covariance matrix. Robertson and Symons (2007) showed that MLFA can be feasible when the number of variables is greater than the number of observations. They assume that the unique variances are positive definite for the existence of the maximum likelihood solution. However, this is not necessarily true with a singular sample covariance matrix.

The first factor may be regarded as openness to diversity. The items loaded highly on this factor indicate that creative employees tend to have diverse interests and diverse ways of thinking. They have up-to-date knowledge regarding the ongoing and upcoming business trends. Furthermore, they have good interpersonal relations with people from different backgrounds. The second factor may be considered as management skill. The items associated with this factor indicate fundamental functions of management, such as planning, organizing, commanding, coordinating, and controlling. Creative employees with a high level of management skill may not only establish a sequence of actions, but also control the work process to produce desirable outputs. The third factor may be regarded as challenge. Creative employees tend to confront a challenge and to take a chance on new tasks. The last factor may be called persistence. Creative employees show strong adherence to their interests in tasks.

\section{Summary and conclusions}

REFA was developed by combining a class of noniterative estimations of unique variances and three widely used estimation methods-ML, GLS, and ULS. An optimal value of the regularization parameter is chosen in such a way that a discrepancy function of ML, GLS, or ULS is minimized. In this approach, unique variances are assumed to be proportional to a noniterative estimate obtained from sample data, thereby reducing the number of parameters for unique variances to one. Estimating one single parameter effectively helps us to fit the factor model to data with a small sample size.

The present article undertook investigations of the performance of three approaches to exploratory factor analysis (MLFA, PCA, and REFA) via analyses of simulated data under various experimental conditions. We summarize the major findings of this study as follows. Regardless of level of communality, REFA generally recovered factor loading parameters better than did MLFA and PCA. The parameter estimates of REFA had smaller mean absolute differences, as well as smaller mean square errors (i.e., closer to true parameters), as compared with those of MLFA and PCA. This superior parameter recovery of REFA over the other approaches was found in most of the sample sizes considered. Although the regularized covariance matrix helps MLFA yield consistent parameter estimates, its relatively poor small-sample behavior is consistent with the literature (MacCallum et al., 1999).

It is worth noting that REFA can also provide stable factor-loading estimates with a singular sample covariance matrix. There are many important practical situations where the sample size could be smaller than the number of observed variables. For instance, in brainimaging studies (e.g., fMRI), the data are very high dimensional (often in the order of millions), and the sample sizes are very small (often in the order of tens). Examples of such data are not limited to neuroimaging data but also abound in face recognition research, signal processing research, DNA microarray research, behavioral genetics research, and so on.

\section{Software notes}

MATLAB programs that carried out the computations reported in this article are available from the author upon request. 


\section{Appendix}

This appendix contains three population covariance matrices used for the simulation study conducted in the present article.

Table 4 High communality

\begin{tabular}{|c|c|c|c|c|c|c|c|c|c|}
\hline \multicolumn{10}{|l|}{1.000} \\
\hline .087 & 1.000 & & & & & & & & \\
\hline .001 & -.070 & 1.000 & & & & & & & \\
\hline .635 & .352 & .072 & 1.000 & & & & & & \\
\hline-.077 & .147 & .639 & .073 & 1.000 & & & & & \\
\hline-.084 & .623 & -.039 & .197 & .212 & 1.000 & & & & \\
\hline-.028 & -.064 & .799 & .046 & .646 & -.024 & 1.000 & & & \\
\hline .047 & -.059 & .746 & .115 & .591 & -.043 & .744 & 1.000 & & \\
\hline .672 & .101 & .194 & .738 & .075 & -.082 & .162 & .232 & 1.000 & \\
\hline .517 & .353 & .101 & .688 & .119 & .234 & .081 & .134 & .614 & 1.000 \\
\hline
\end{tabular}

Table 5 Wide communality

\begin{tabular}{|c|c|c|c|c|c|c|c|c|c|}
\hline \\
\hline \multicolumn{10}{|l|}{$\begin{array}{l}1.000 \\
.347\end{array}$} \\
\hline-.071 & -.010 & 1.000 & & & & & & & \\
\hline .208 & .093 & .540 & 1.000 & & & & & & \\
\hline .082 & .286 & .190 & .054 & 1.000 & & & & & \\
\hline .094 & .172 & .215 & .139 & .325 & 1.000 & & & & \\
\hline .271 & .396 & .094 & .066 & .617 & .338 & 1.000 & & & \\
\hline .051 & .148 & .747 & .510 & .404 & .321 & .353 & 1.000 & & \\
\hline .008 & .202 & .150 & .014 & .486 & .256 & .483 & .316 & 1.000 & \\
\hline .011 & .250 & .132 & -.022 & .593 & .304 & .593 & .342 & .488 & 1.000 \\
\hline
\end{tabular}

Table 6 Low communality

\begin{tabular}{|c|c|c|c|c|c|c|c|c|c|}
\hline \multicolumn{10}{|l|}{1.000} \\
\hline-.008 & 1.000 & & & & & & & & \\
\hline .186 & .064 & 1.000 & & & & & & & \\
\hline .245 & -.008 & .231 & 1.000 & & & & & & \\
\hline .179 & .119 & .193 & .220 & 1.000 & & & & & \\
\hline .038 & .341 & .093 & .048 & .142 & 1.000 & & & & \\
\hline-.019 & .227 & .162 & -.019 & .060 & .186 & 1.000 & & & \\
\hline .276 & .065 & .252 & .338 & .271 & .118 & -.006 & 1.000 & & \\
\hline .198 & .027 & .184 & .243 & .188 & .068 & -.007 & .282 & 1.000 & \\
\hline-.011 & .078 & .149 & -.009 & .021 & .061 & .222 & -.024 & -.012 & 1.000 \\
\hline
\end{tabular}




\section{References}

Bickel, P. J., \& Li, B. (2006). Regularization in statistics. Test, 15, 271-303. Cohen, J. (1988), Statistical power analysis for the behavioral sciences (2nd ed.). Hillsdale, NJ: Erlbaum.

Colibazzi, T., Zhu, H., Bansal, R., Schultz, R. T., Wang, Z., \& Peterson B. S. (2008). Latent volumetric structure of the human brain: Exploratory factor analysis and structural equation modeling of gray matter volumes in healthy children and adults. Human Brain Mapping, 29, 1302-1312.

Curran, P. J., West, S. G., \& Finch, J. F. (1996). The robustness of test statistics to nonnormality and specification error in confirmatory factor analysis. Psychological Methods, 1, 16-29.

De Winter, J. C. F, Dodou, D., \& Wieringa, P. A. (2009). Exploratory factor analysis with small sample sizes. Multivariate Behavioral Research, 44, 147-181.

Fabrigar, L. R., Wegener, D. T., MacCallum, R. C., \& Strahan, E. J. (1999). Evaluating the use of exploratory factor analysis in psychological research. Psychological Methods, 4, 272-299.

Finkbeiner, C. T., \& Tucker, L. R. (1982). Approximate uniqueness estimates for singular correlation matrices. Psychometrika, 47, $517-521$

Fleishman, A. I. (1978). A method for simulating non-normal distributions. Psychometrika, 43, 521-532.

Flora, D. B., \& Curran, P. J. (2004). An empirical evaluation of alternative methods of estimation for confirmatory factor analysis with ordinal data. Psychological Methods, 9, 466-491.

Hägglund, G. (1982). Factor analysis by instrumental variables methods. Psychometrika, 47, 209-222.

Hoerl, A. F., \& Kennard, R. W. (1970). Ridge regression: Biased estimation for nonorthogonal problems. Technometrics, 12, 55-67.

Ihara, M., \& Kano, Y. (1986). A new estimator of the uniqueness in factor analysis. Psychometrika, 51, 563-566.

Jolliffe, I. T. (2002). Principal component analysis. New York: Springer.

Jöreskog, K. G., \& Sörbom, D. (1993). LISREL 8. Mooresville, IN: Scientific Software.

Jung, S., \& Takane, Y. (2008). Regularized exploratory factor analysis. In K. Shigemasu, A. Okada, T.Imaizumi, \& T. Hoshino (Eds.), New trends in psychometrics (pp. 141-149). Tokyo: University Academic Press.

Lee, S. (2008). A conceptual and empirical approach to creativity in industrial organizations. Korean Journal of Industrial and Organizational Psychology, 21, 151-178.
Legendre, P., \& Legendre, L. (1998). Numerical ecology (2nd English ed.). Oxford: Elsevier.

MacCallum, R. C., \& Austin, J. T. (2000). Applications of structural equation modeling in psychological research. Annual Review of Psychology, 51, 201-226.

MacCallum, R. C., Widaman, K. F., Zhang, S., \& Hong, S. (1999). Sample size in factor analysis. Psychological Methods, 4, 84-89.

Mason, C. H., \& Perreault, W. D., Jr. (1991). Collinearity, power, and interpretation of multiple regression analysis, Journal of Marketing Research, 28, 268-280.

Maxwell, S. E., \& Delaney, H. D. (1990). Designing experiments and analyzing data. Belmont, CA: Wadsworth.

Micceri, T. (1989). The unicorn, the normal curve, and other improbable creatures. Psychological Bulletin, 105, 156-166.

Mood, A. M., Graybill, F. A., \& Boes, D. C. (1973), Introduction to the theory of statistics ( $3^{\text {rd }}$ ed.). New York: McGraw-Hill.

O'Connor, B. P. (2000). SPSS and SAS programs for determining the number of components using parallel analysis and Velicer's MAP test. Behavior Research Methods, Instruments, \& Computers, 32, 396-402.

Robertson, D., \& Symons, J. (2007). Maximum likelihood factor analysis with rank-deficient sample covariance matrices. Journal of Multivariate Analysis, 98, 813-828.

Spearman, C. (1904). General intelligence, objectively determined and measured. American Journal of Psychology, 15, 201-293.

Tanaka, J. S. (1987). How big is big enough? Sample size and goodness-of-fit in structural equation models with latent variables. Child Development, 58, 135-146.

Tien, A. Y., Eaton, W. W., Schlaepfer, T. E., McGilchrist, I. K., Menon, R., Powers, R., . . . Pearlson, G. D. (1996). Exploratory factor analysis of MRI brain structure measures in schizophrenia. Schizophrenia Research, 19, 93-101.

Wijsman, R. A. (1959). Applications of a certain representation of the Wishart matrix. Annals of Mathematical Statistics, 30, 597601.

Yates, A. (1984) Multivariate exploratory data analysis: A perspective on exploratory factor analysis. Albany: State University of New York Press.

Yuan, K.-H., \& Chan, W. (2008). Structural equation modeling with near singular covariance matrices. Computational Statistics and Data Analysis, 52, 4842-4858. 